\title{
Nasal Colivelin Treatment Ameliorates Memory Impairment Related to Alzheimer's Disease
}

\author{
Marina Yamada',2,3, Tomohiro Chiba*, ${ }^{*, 2}$, Jumpei Sasabe ${ }^{1,2}$, Kenzo Terashita', Sadakazu Aiso' \\ and Masaaki Matsuoka*,I,2 \\ 'Department of Anatomy, KEIO University School of Medicine, Tokyo, Japan; ${ }^{2}$ Department of Cell Biology and Neurosciences, KEIO University \\ School of Medicine, Tokyo, Japan
}

\begin{abstract}
Humanin $(\mathrm{HN})$ and its derivatives, such as Colivelin (CLN), suppress neuronal death induced by insults related to Alzheimer's disease (AD) by activating STAT3 in vitro. They also ameliorate functional memory impairment of mice induced by anticholinergic drugs or soluble toxic amyloid- $\beta(A \beta)$ in vivo when either is directly administered into the cerebral ventricle or intraperitoneally injected. However, the mechanism underlying the in vivo effect remains uncharacterized. In addition, from the standpoint of clinical application, drug delivery methods that are less invasive and specific to the central nervous system (CNS) should be developed. In this study, we show that intranasally (i.n.) administered CLN can be successfully transferred to CNS via the olfactory bulb. Using several behavioral tests, we have demonstrated that i.n. administered CLN ameliorates memory impairment of AD models in a dose-responsive manner. Attenuation of AD-related memory impairment by HN derivatives such as CLN appears to be correlated with an increase in STAT3 phosphorylation levels in the septohippocampal region, suggesting that anti-AD activities of HN derivatives may be mediated by activation of STAT3 in vivo as they are in vitro. We further demonstrate that CLN treatment inhibits an $A \beta$ induced decrease in the number of choline acetyltransferase (ChAT)-positive neurons in the medial septum. Combined with the finding that HN derivatives upregulate mRNA expression of neuronal ChAT and vesicular acetylcholine transporter (VAChT) in vitro, it is assumed that CLN may ameliorate memory impairment of AD models by supporting cholinergic neurotransmission, which is at least partly mediated by STAT3-mediated transcriptional upregulation of ChAT and VAChT.
\end{abstract}

Neuropsychopharmacology (2008) 33, 2020-2032; doi:I0.1038/sj.npp. I 30159I; published online I0 October 2007

Keywords: Alzheimer's disease; humanin; activity-dependent neurotrophic factor; Colivelin; memory impairment

\section{INTRODUCTION}

Development of effective Alzheimer's disease (AD) therapy is urgently required because AD currently affects nearly $2 \%$ of the population in industrialized countries (Mattson, 2004). AD is characterized clinically by progressive memory impairment and pathologically by senile plaques, neurofibrillary tangles, and neuronal death. Several lines of evidence have also revealed that dysfunction in the cholinergic system of the basal forebrain is involved in the pathogenesis of $\mathrm{AD}$ (Bartus et al, 1982; Coyle et al, 1983). Progressive cholinergic denervation in the cerebral cortex as well as decreased levels of acetylcholine (ACh) and choline acetyltransferase (ChAT) have been reported to be associated with $\mathrm{AD}$-relevant cognitive impairment (Bartus

* Correspondence: Drs T Chiba and M Matsuoka, Department of Cell Biology and Neurosciences, KEIO University School of Medicine, 35 Shinanomachi, Shinjuku-ku, Tokyo 160-8582, Japan, Tel: +8I 35363 8427, Fax: +81 35363 8428, E-mails: chibat@sc.itc.keio.ac.jp, sakimatu@sc.itc.keio.ac.jp

${ }^{3}$ Japan Society for the Promotion of Science Research Fellow. Received 14 February 2007; revised 21 August 2007; accepted 26 August 2007 et al, 1982; Coyle et al, 1983). A mutually aggravating relationship between cholinergic dysfunction and pathological changes in $\mathrm{AD}$ has also been reported. Dysfunction of cholinergic neurotransmission may contribute to the development of amyloid plaque pathology (Caccamo et al, 2006) and toxic soluble amyloid- $\beta$ (A $\beta)$ disrupts the cholinergic neurotransmission (Bales et al, 2006). Moreover, acetylcholinesterase (AChE) inhibitors, such as donepezil, improve cognitive function in $\mathrm{AD}$ patients to some extent (Lane et al, 2004; Petersen et al, 2005; Winblad et al, 2006). Taken altogether, supporting cholinergic neurotransmission is deemed to be a promising strategy for $A D$ therapy, especially for patients in the early stage of the disease, when massive and irreversible brain atrophy has not yet taken place.

Humanin (HN), originally identified from an occipital lobe of an $\mathrm{AD}$ patient, abolishes neuronal cell death induced by various familial $\mathrm{AD}$ (FAD) causative genes and $\mathrm{A} \beta$ (Hashimoto et al, 2001a, b; Nishimoto et al, 2004). Colivelin (CLN), the strongest $\mathrm{HN}$ derivative so far developed, is a fusion peptide composed of a potent $\mathrm{HN}$ derivative named AGA-(C8R)HNG17 attached to the C terminus of activitydependent neurotrophic factor (ADNF) (Brenneman and 
Gozes, 1996; Brenneman et al, 1998; Chiba et al, 2004, 2005, 2006; Matsuoka et al, 2006). Our earlier studies indicated that $\mathrm{HN}$ and CLN suppressed AD-relevant neuronal death by activating STAT3 in vitro (Chiba et al, 2005; Hashimoto et al, 2005; Matsuoka et al, 2006). It has been also demonstrated in vivo that $\mathrm{HN}$ derivatives, including CLN, suppress spatial working memory impairment induced by blockers to the cholinergic system or intracerebroventricular (i.c.v.) injection of toxic $\mathrm{A} \beta$ peptides (Mamiya and Ukai, 2001; Krejcova et al, 2004; Chiba et al, 2005). Considering that both insults cause memory impairment in vivo by inducing the dysfunction of the cholinergic system without significant neuronal death, HN derivatives are likely to support cognitive function by upregulating the cholinergic neurotransmission in vivo. However, the molecular mechanism underlying this activity remains unknown.

From the standpoint of clinical applications for $\mathrm{HN}$ derivatives, it is also important to establish drug delivery methods that are less invasive and central nervous system (CNS) specific to administer CLN. Drugs for AD must be safely and continuously used over a long period, and i.c.v. or i.p. injection is not appropriate. Intranasal (i.n.) administration is one of the less invasive delivery methods for peptide drugs (Illum, 2000; Thorne et al, 2004). It bypasses the blood-brain barrier (BBB), which otherwise restricts entrance of molecules from the bloodstream to the CNS. A number of neurotrophic peptides - including vasoactive intestinal peptide, ADNF, and insulin-like growth factor-1-have been successfully delivered to the brain or the CSF by i.n. administration (Gozes et al, 1996, 2000; Thorne et al, 2004). Given that highly lipophilic small peptides can be relatively easily delivered to the CNS by i.n. administration, $\mathrm{HN}$ derivatives such as CLN may be efficiently transported to the CNS by this method.

In this study, we demonstrate the therapeutic efficacy of i.n. CLN treatment compared with those of i.n. ADNF or AGA-(C8R)HNG17 using two types of AD models: anticholinergic drugs (cholinotoxin)-induced models (Chiba et al, 2005) and a repetitive $\mathrm{A} \beta$ injection model (Yamada et al, 2005). We also characterize the molecular mechanism underlying CLN- or AGA-(C8R)HNG17-mediated support of cognitive function in terms of upregulation of the cholinergic neurotransmission, putatively mediated by STAT3.

\section{METHODS}

\section{Peptides and Materials}

Colivelin (CLN, SALLRSIPAPAGASRLLLLTGEIDLP), ADNF (SALLRSIPA), and AGA-(C8R)HNG17 (PAGASRLLLLTGEIDLP) were synthesized as described previously (Chiba et al, 2005). A rabbit polyclonal anti-HN antibody (PO4) was described previously (Tajima et al, 2002). A rabbit polyclonal anti-ADNF antibody was raised against synthetic ADNF. Affinity-purified polyclonal antibody against ChAT was purchased from Chemicon (Temecula, CA). A rabbit polyclonal antibody against phospho-STAT3 $\left(\mathrm{Tyr}^{705}\right)$ and a rabbit monoclonal antibody against total STAT3 (79D7) were from Cell Signaling Technology (Beverly, MA). A $\beta 25-$ 35 was purchased from Peptide Institute (Osaka, Japan).
Scopolamine hydrobromide and 3-quinuclidinyl benzilate (3-QNB) were from Sigma (St Louis, MO). Other reagents used in the study were all commercially available.

\section{Animals and Treatments}

This study was conducted in accordance with the Policies on the Use of Animals and Humans in Neuroscience Research, the Society for Neuroscience and Guideline for the Care and Use of Laboratory Animals of KEIO University School of Medicine. All experimental procedures were approved by the Institutional Animal Experiment Committee at KEIO University.

CD-1 (ICR) mice at the age of 7 weeks were purchased from Charles River Japan Inc. (Kanagawa, Japan). Animals were housed in a specific pathogen-free animal facility as described previously (Kawasumi et al, 2004; Yamada et al, 2005). Cholinotoxin models were described previously (Chiba et al, 2005). Briefly, CD-1 mice at the age of 8 weeks were given $10 \mu \mathrm{l}$ vehicle (sterile deionized distilled water (ddw) containing 5\% sefsol and 20\% isopropanol) i.n. (Gozes et al, 1996, 2000) with or without indicated amounts of synthetic CLN peptides twice, at $24 \mathrm{~h}$ and $30 \mathrm{~min}$ before the Y-maze test (YM), as depicted in Figure 2a. Scopolamine $(1 \mathrm{mg} / \mathrm{kg})$ dissolved in $0.2 \mathrm{ml}$ sterile saline was subcutaneously (s.c.) injected at $30 \mathrm{~min}$ before YM whereas 3-QNB $(0.5 \mathrm{mg} / \mathrm{kg})$ dissolved in $0.2 \mathrm{ml}$ sterile ddw containing $20 \%$ methanol was i.p. injected at $15 \mathrm{~min}$ before YM to induce amnesia.

The $\mathrm{AD}$ mouse model by repetitive i.c.v. injection of $\mathrm{A} \beta$ was described previously (Yamada et al, 2005). Briefly, CD1 mice at the age of 8 weeks were stereotaxically implanted with cannulas (C315GS-4; PlasticOne Inc., Roanoke, VA) in the left lateral ventricle (anterior-posterior, $+0.3 \mathrm{~mm}$; lateral, $1.0 \mathrm{~mm}$; and horizontal, $3.0 \mathrm{~mm}$ from the bregma). Ten days after implantation, animals were randomly assigned to the control group, $\mathrm{A} \beta$-injected group, or the $\mathrm{A} \beta$-injected plus CLN-treated group, receiving i.c.v. injection of either $3 \mu \mathrm{l}$ sterile ddw or $1 \mathrm{nmol} \mathrm{A} \beta 25-35$ peptide in $3 \mu \mathrm{ddw}$, every other day for 3 weeks (10 times) together with i.n. administration of $10 \mu \mathrm{l}$ vehicle with or without $1 \mathrm{nmol}$ CLN once a day for 3 weeks, as depicted in Figure 7a.

\section{Behavioral Tests}

An open field test (OF) was performed as described previously (Kawasumi et al, 2004; Yamada et al, 2005) to habituate the mice to following experimental conditions. YM was performed as described previously (Yamada et al, 2005). Briefly, the apparatus for YM is made of three gray plastic arms $(40 \mathrm{~cm}$ long, $12 \mathrm{~cm}$ high, $3 \mathrm{~cm}$ wide at the bottom, and $10 \mathrm{~cm}$ wide at the top). Mice were allowed to explore the maze freely for $8 \mathrm{~min}$. Spontaneous alternation percentage (SA\%), an index of spatial working memory, was defined as the ratio of the arm choices that differed from the previous two choices (correct choices) to total choices during the run (total arm entry, 2).

A memory assessment version of the elevated-plus maze (EPM) was performed as described (Itoh et al, 1991). The testing apparatus, set at $100 \mathrm{~cm}$ above the floor, was made of four arms crossed perpendicularly at the center. Two arms were open $(50 \times 10 \mathrm{~cm}$ gray plastic floor plates without 
walls), while the other two were closed (with $10 \mathrm{~cm}$ high gray plastic walls). Mice were individually placed at the end of the open arm and allowed to explore the maze freely for $90 \mathrm{~s}$. The test was performed twice at a 24 -h interval without any specific restriction. The first session was performed after scopolamine treatment by the same procedure as that depicted in Figure 2a. Examined parameters were (1) transfer latency, defined as the time elapsed until the first entry to the closed arms; (2) duration of the first stay in a closed arm, defined as the time from first entry to a closed arm to first escape from the arm; and (3) cumulative time spent in open/closed arms.

A passive avoidance (PA) task was performed as described (Jhoo et al, 2004). The PA apparatus consisted of two chambers separated with a gray plastic guillotine door; one $(11 \mathrm{~cm}$ long $\times 5 \mathrm{~cm}$ wide, with $13 \mathrm{~cm}$ walls) was illuminated and the other $(25 \mathrm{~cm}$ long $\times 25 \mathrm{~cm}$ wide, with $20 \mathrm{~cm}$ walls) was darkened with a constant current shock generator. The test was performed twice at a $24-\mathrm{h}$ interval without any specific restriction. In an acquisition trial (day 1 ), each mouse with or without s.c. scopolamine administration $(0.5 \mathrm{mg} / \mathrm{kg})$ was confined in the illuminated chamber for $15 \mathrm{~s}$ and then the door was opened for the mouse to move freely into the dark chamber. Soon after the mice entered the dark chamber, the door was closed and an inescapable electric shock $(0.3 \mathrm{~mA}, 3 \mathrm{~s}$, once) was delivered through the floor grid. Each mouse was again tested in the apparatus (day 2, a retention trial) $24 \mathrm{~h}$ later. Entry latency (or step-through latency) defined as elapsed time until the mice entered into the dark chamber (maximum $300 \mathrm{~s}$ ) was measured as an index of contextual memory.

A radial arm maze (RAM) task was performed as described (Kawasumi et al, 2004). Eight arms were connected radially to an octagonal center platform, on which a transparent guillotine door was placed to retain animals on the platform. Each arm was made of a $40 \times 7 \mathrm{~cm}$ gray plastic floor without walls. The apparatus was elevated at $100 \mathrm{~cm}$ from the floor. Mice were deprived of chow for $12 \mathrm{~h}$ before the test; to motivate them, small chow pellets $(10 \mathrm{mg})$ were placed at the end of each arm. Mice were first confined at the central octagon individually for $10 \mathrm{~s}$, and then were allowed to explore the maze freely until they finished choosing all eight arms. The task ended when the tested mouse chose all arms (maximum $300 \mathrm{~s}$ ). Examined parameters were (1) total time required to finish the task (Total time) and (2) working memory error percentages in the first eight arm entries (Error \% in the first eight entries).

\section{Biochemical Measurements of Serum ALT/GPT, AST/GOT, and CRE}

To examine the acute side effects of CLN, male CD-1 mice at the age of 8 weeks were singly intravenously (i.v.) administered with either $0.1 \mathrm{ml}$ saline or saline containing $200 \mathrm{nmol}$ CLN. Serum of the treated mice was sampled for biochemical analysis of alanine aminotransferase (ALT, or GPT), aspartate aminotransferase (AST, or GOT), and creatinin (CRE) by Fuji Dri-Chem 3000 (Fuji-Film, Tokyo, Japan, $N=3$ ), $6 \mathrm{~h}$ after the injection. For subacute side effect analysis, male CD-1 mice at the age of 8 weeks were i.n. administered with $10 \mu \mathrm{l}$ vehicle or vehicle containing $5 \mathrm{nmol}$ CLN once a day for 1 week.

\section{Histological Analysis}

Immunohistochemical (IHC) analysis was performed as described previously (Chiba et al, 2005; Yamada et al, 2005). Under general anesthesia with diethyl ether, mice were transcardially perfused with phosphate-buffered saline (PBS), and fixed by ethanol containing 5\% acetic acid. Paraffin-embedded sections at $10 \mu \mathrm{m}$ were prepared and were soaked with the appropriate primary antibodies (anti-ChAT antibody, 1:50 dilution; anti-ADNF antibody, $1: 50 ; \mathrm{PO} 4,1: 100)$ and the appropriate second antibody. Immunoreactivity was visualized with the $A B C$ method (for ChAT, Vectastain Elite Kit, Vector, CA, USA) or the FITClabeled anti-rabbit secondary antibody (for anti-ADNF antibody and PO4). ChAT-immunoreactive neurons in the medial septa of five coronal sections were counted, and the averages of the total numbers of ChAT-positive neurons in three mice per treatment group $(N=3)$ were compared (Yamada et al, 2005).

\section{Immunoblot Analysis of STAT3 Phosphorylation}

After i.n. treatment of several peptides, mice were transcardially perfused with PBS. The olfactory bulbs and hippocampuses of the mice were then sampled and frozen by liquid nitrogen. For immunoblot analysis, brain samples were lysed in a lysis buffer $(50 \mathrm{mM}$ Tris- $\mathrm{HCl}(\mathrm{pH} 7.4)$, $150 \mathrm{mM} \mathrm{NaCl}, 1 \%$ Triton X-100, protease inhibitors, $1 \mathrm{mM}$ EDTA, phosphatase inhibitor cocktails 1 and 2 (Sigma)). Samples were then subjected to normal SDS-PAGE and blotted onto polyvinylidene fluoride membranes. The membranes were soaked with anti-phospho-STAT3 antibody $(1: 1000)$ and then with horseradish peroxidaselabeled anti-rabbit IgG antibody (1:5000) (Bio-Rad Laboratories, Hercules, CA, USA). Immunoreactive bands were detected with ECL Western Blotting Detection Reagents (Amersham Bioscience, Uppsala, Sweden). Total STAT3 was visualized with the specific antibody $(1: 3000)$ on the identical membrane after stripping of anti-p-STAT3 antibody. Densitometric analysis of the results was carried out with NIH Image (ver. 1.62) software.

\section{Assessment of mRNA Levels (RT-PCR)}

Total RNA was extracted from PC12 cells $\left(1.2 \times 10^{5}\right.$ cells per well in a six-well plate, cultured in DMEM containing $10 \%$ fetal bovine serum) that had been treated with or without 100 pM of CLN, ADNF, or AGA-(C8R)HNG17 by the TRIzol reagent (Invitrogen, Carlsbad, $\mathrm{CA}$ ) according to the manufacturer's protocol. First-strand cDNAs were synthesized from $1 \mu \mathrm{g}$ total RNA using Omniscript reverse transcriptase according to the manufacturer's protocol (Quiagen, Valencia, CA). PCR amplification with Taq DNA polymerase (Sigma) was performed under the following condition of denaturation at $94^{\circ} \mathrm{C}$ for $30 \mathrm{~s}$, annealing at $60^{\circ} \mathrm{C}$ for $30 \mathrm{~s}$, and extension at $72^{\circ} \mathrm{C}$ for $90 \mathrm{~s}$, repeated 25 cycles for glyceraldehyde-3-phosphate dehydrogenase (GAPDH) and 35 cycles for ChAT and acetylcholine transporter (VAChT). The following sequences were used for forward and reverse primer: ChAT, forward 5'-GG GTGATCTGTTCACTCAGTTGAG- ${ }^{\prime}$, and reverse $5^{\prime}$-CTCTG GTAAAGCCTGTAGTAAGCC-3’ (669 bp); VAChT, forward 
$5^{\prime}$-AGCGGGCCTTTCATTGATCG- ${ }^{\prime}$, and reverse $5^{\prime}$-GGCGC ACGTCCACCAGGAAGG-3' (814 bp); and GAPDH, forward $5^{\prime}$-GAACATCATCCCTGCATCC-3', and reverse 5'-GCTTCA CCACCTTCTTGATG-3' (179 bp).

\section{AChE Activity Assay (Ellman's Method)}

The reaction mixture was prepared as follows: phosphate buffer containing 5,5'-dithio-bis-(2-nitrobenzoic acid) (DTNB, $1 \mu \mathrm{g} / \mathrm{ml}$, Sigma), $0.05 \%$ acetylthiocholine (Sigma), and indicated concentrations of tacrine (Sigma) or CLN (total volume; $100 \mu \mathrm{l}$ ). The mixture was incubated for $15 \mathrm{~min}$ at $37^{\circ} \mathrm{C}$. AChE (acetylcholinesterase; $1 \mu \mathrm{l}$, final; $0.01 \mathrm{U} / \mu \mathrm{l}$, purified from electric eel, Sigma) was then added to the mixture and further incubated for $15 \mathrm{~min}$. Reaction was stopped by rapid icing. The absorbance at $416 \mathrm{~nm}$ of the mixture represented the AChE activity.

\section{Statistics}

All values in the figures of the in vitro study indicate means \pm standard deviation (SD), and the values of the in vivo study indicate means \pm standard errors of means (SEM). Statistical analysis was performed with one-way analysis of variance (ANOVA), in which $\alpha$ value was set at 0.05 , followed by Tukey/Kramer's post hoc multiple comparison test unless specifically indicated in the figure legends. Statistical analysis of RAM data was performed with two-way repeated-measures ANOVA followed by Tukey/Kramer's post hoc test. All data were analyzed using StatView (ver.5.0.1) software.

\section{RESULTS}

\section{Intranasally Administered CLN is Transported to CNS via the Olfactory Bulb}

In our earlier study, we reported that i.c.v. injection of CLN almost completely antagonized $\mathrm{A} \beta$ neurotoxicity in vivo (Chiba et al, 2005). To develop a less invasive drug delivery system for CLN, we investigated whether i.n. administered CLN was transported to the CNS by immunofluorescence (IF)-based visualization of CLN in the olfactory bulb. Coronal sections of paraffin-embedded brains of mice with i.n. administration of vehicle or CLN were soaked with antiADNF antibody that recognizes ADNF domain of CLN and then visualized by FITC-labeled secondary antibody. IF intensity was significantly higher in the medial regions of the olfactory bulbs of the CLN-treated mice than those of the vehicle-treated control mice (Figure 1a). We also observed granular increase in IF intensity in the cerebral cortices and an overall increase in IF intensity around the optic chiasms. A similar IF staining pattern was obtained with anti-HN antibody (PO4) (Figure $1 \mathrm{~b}$ ). We confirmed not only that treatment with a secondary antibody alone did not result in similar IF staining but also that the immunoreactivity to anti-ADNF antibody was preabsorbed by synthetic ADNF and that to PO4 was preabsorbed by synthetic HN peptide (Figure 1c). Considering that the two antibodies used here recognize distinct epitopes corresponding to ADNF (N-terminal) and AGA-(C8R)HNG17 (C-terminal) portions of CLN, it is highly likely that i.n. administered CLN reached the brain regions stained with both antibodies. Although detailed mechanisms of CLN's delivery to the CNS remain to be examined, it is suggested that i.n. administered CLN may be delivered highly specifically to the CNS through some cerebral nerves, including the olfactory and the optic nerve (Illum, 2000; Thorne et al, 2004).

\section{Intranasally Administered CLN Ameliorates Scopolamine-Induced Spatial Working Memory Impairment in a Y-Maze Test}

Dysfunction of the cholinergic system in the basal forebrain has been implicated in AD-relevant memory impairment (Bartus et al, 1982; Coyle et al, 1983). In accordance, administration of cholinotoxins such as scopolamine, 3-QNB, and ethylcholine aziridium (AF64A) has been reported to induce memory impairment in both rodents and humans (Fisher et al, 1989; Krejcova et al, 2004). We examined the effect of i.n. administration of CLN on scopolamine-induced working memory impairment in a YM (Figure 2a and b). As reported in earlier studies (Mamiya and Ukai, 2001; Chiba et al, 2005), s.c. administration of scopolamine significantly reduced the SA\%, an index of the spatial working memory in YM, suggesting that scopolamine impaired the spatial working memory. Intranasal administration of CLN significantly attenuated scopolamine-induced decrease in SA\% in a dose-dependent manner (Figure $2 \mathrm{~b}$ ). Intranasally administered CLN itself did not significantly affect SA\% of mice without scopolamine treatment (Figure 2c), supporting the idea that CLN antagonized scopolamine-induced memory impairment.

\section{Comparison of the Pharmacological Effect of CLN via Various Drug Delivery Routes}

To assess the advantages of i.n. administration, we next compared the effect of i.n. CLN treatment with those of intraperitoneal (i.p.) or s.c. administration of CLN at the same dose ( $1 \mathrm{nmol}$ per administration, twice at a $24-\mathrm{h}$ interval) in the scopolamine model (Figure 2d). Scopolamine again significantly reduced SA\% in YM. Neither i.p. nor s.c. administration of CLN ameliorated scopolamineinduced decrease in SA\%, while i.n. treatment of CLN at the same dose did so in a statistically significant manner (Figure 2d), suggesting that i.n. administration may be a more efficient drug delivery route for CLN to the CNS.

\section{Assessment of the Pharmacological Effect of CLN by Other Behavioral Tests Evaluating Distinct Memory Paradigms}

To confirm the efficacy of CLN, we next examined the effect of i.n. administered CLN on scopolamine-induced memory impairment using other behavioral tests. We first performed 'EPM', a widely used test for anxiety, which can also be used as a fear-motivated spatial learning test, as reported in an earlier study (Itoh et al, 1991). This test consists of two identical sessions, separated by a 24 -h interval. In the second session, mice with normal memory that learned the EPM apparatus in the first session tend to avoid open arms 
a
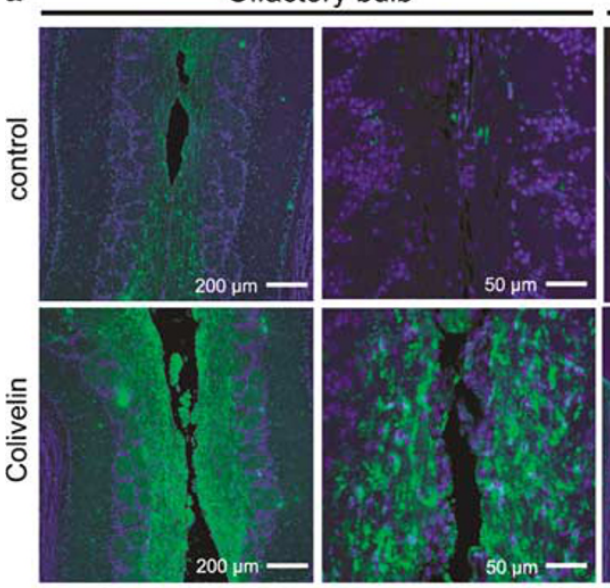

Cerebral cortex
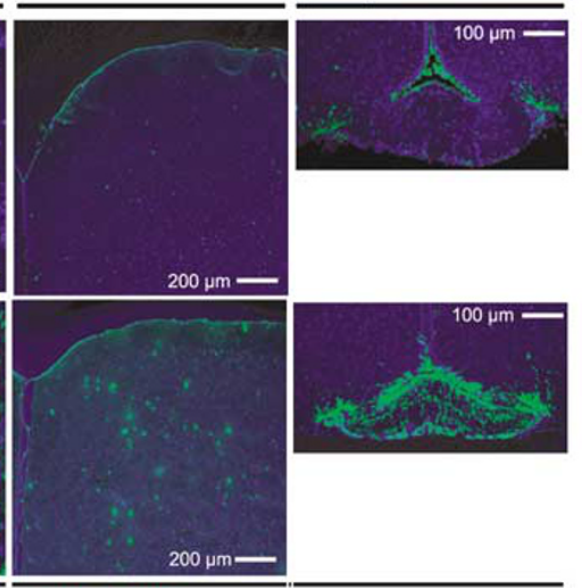

TO-PRO-3 / anti-ADNF

b

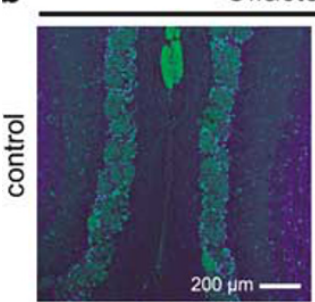

Olfactory bulb

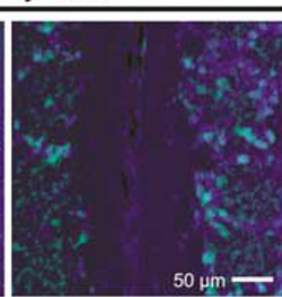

Cerebral cortex
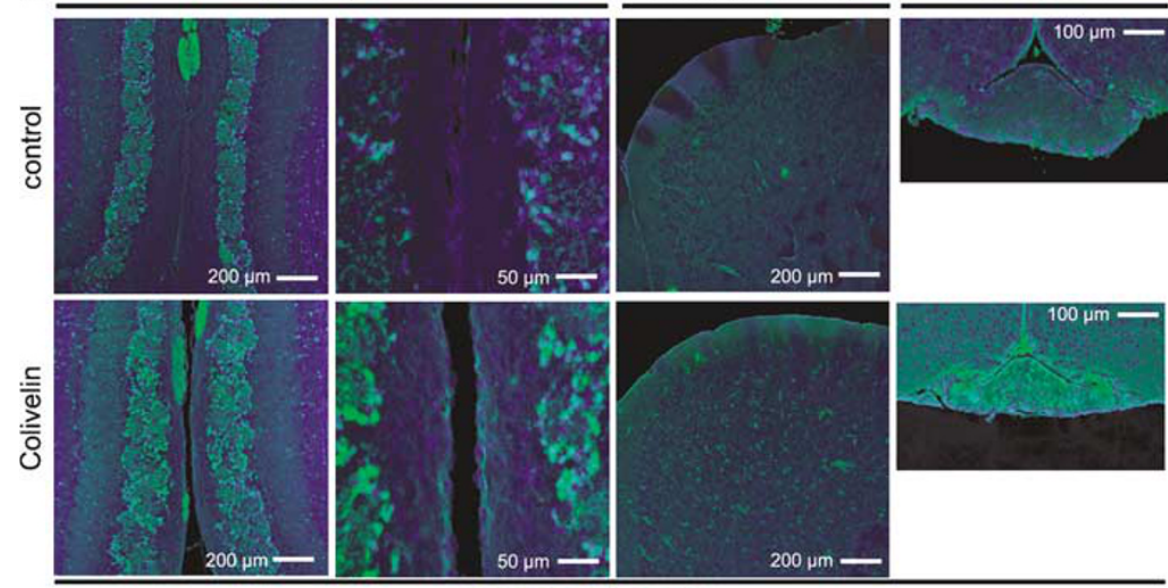

TO-PRO-3 / PO4

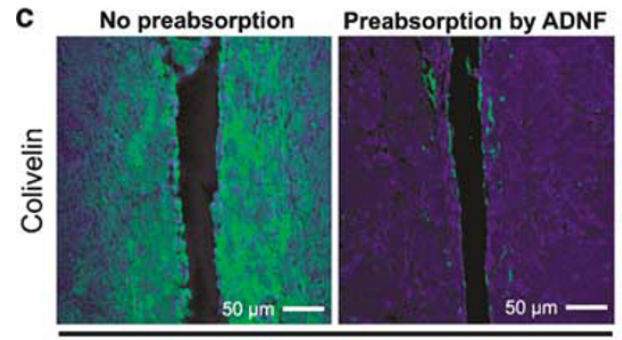

TO-PRO-3 I anti-ADNF

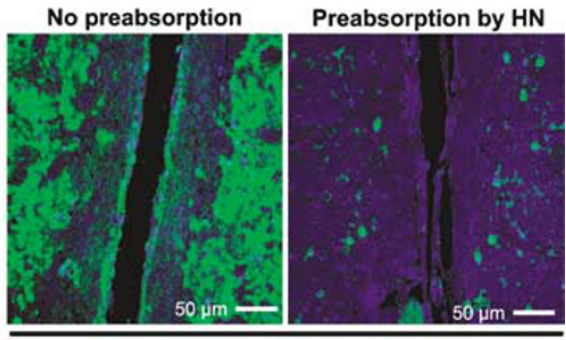

TO-PRO-3 / PO4

Figure I CNS delivery of Colivelin (CLN) by i.n. administration. Intranasally administered CLN in the brain was immunohistochemically visualized. CLN $(5 \mathrm{nmol})$ was i.n. administered to mice $30 \mathrm{~min}$ before being killed. Paraffin-embedded coronal sections of the olfactory bulbs (left panel, bar: $200 \mu \mathrm{m}$; right panel, bar: $50 \mu \mathrm{m}$ ), the cerebral cortices (primary motor cortex, bar: $200 \mu \mathrm{m}$ ), or the optic chiasms (bar: $100 \mu \mathrm{m})$ of vehicle-treated and CLN-treated mice were immunohistochemically stained with an anti-activity-dependent neurotrophic factor (ADNF) antibody (a, green) or an antibody raised against humanin $(\mathrm{HN})$ peptide (PO4) (b, green) to detect administered CLN peptide. Nuclear staining was performed with TO-PRO-3 (blue). Preincubation (or preabsorption) of the anti-ADNF antibody and PO4 with their synthetic antigenic peptides (ADNF or HN at $10 \mu \mathrm{M}$ ) almost completely diminished IF intensity observed in the olfactory bulbs of mice with i.n. administration of CLN (c).

because mice fear high places. Accordingly, 'transfer latency' from the open arm to the closed arm and 'time spent in open arms' were significantly shortened (Figure 3a and c) while 'duration of the first stay in closed arm' and 'time spent in closed arms' were significantly prolonged (Figure $3 \mathrm{~b}$ and $\mathrm{c}$ ) in the control mice. In clear contrast, there were no significant changes in the four examined parameters in scopolamine-treated mice, indicating that scopolamine treatment completely inhibited memory acquisition in the first session (Figure 3a-d). Mice treated with scopolamine together with i.n. CLN showed memory acquisition to an extent similar to the control mice (Figure 3a-d), indicating that CLN antagonized scopolamineinduced inhibition of memory acquisition in the EPM.

We also performed a PA task to evaluate contextual memory (Figure $4 \mathrm{a}$ ). In the acquisition trial (day 1), there 
a

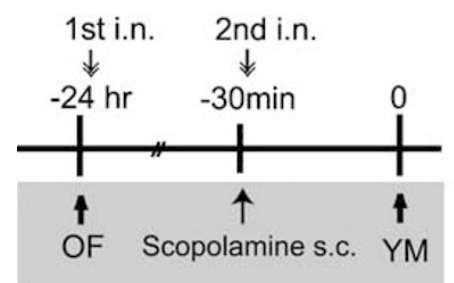

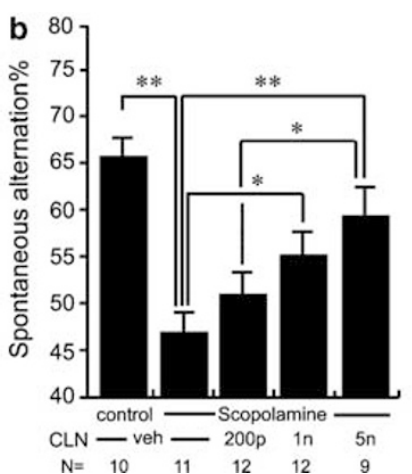

Figure 2 Effect of i.n. administered Colivelin (CLN) on a cholinotoxin model in a Y-maze test (YM). The design of the experiment is depicted in (a). Open field (OF) test was performed to habituate mice to the experimental conditions. We examined the effect of i.n. administered CLN on scopolamine (I.0 mg/ $\mathrm{kg}$, s.c.)-induced working memory impairment in YM (b). Indicated doses of CLN were administered per application. Spontaneous alternation percentages $(\mathrm{SA} \%)$ of each treatment group are shown as means $\pm \mathrm{SEM}$. ANOVA revealed a significant effect of treatments on $\mathrm{SA} \%(\mathrm{~F}(4,49)=8.730 ; \mathrm{P}<0.000 \mathrm{I})$. $* P<0.05$, ** $P<0.0$ I by Fisher's PLSD multiple comparison test. Effect of i.n. CLN itself on SA\% was also examined (c). There was no significant effect of the CLN dose on SA\% $\left(F_{(3,48)}=0.287 ; P=0.8344\right)$. 'n.s.' means 'not significant'. We also compared the effects of CLN via different delivery routes (i.p., s.c., or i.n.) at the same dose (I nmol/application, two times as depicted in (a)) on scopolamine-induced memory impairment (d). ANOVA revealed a significant effect of treatments on SA\% $\left(F_{(4,85)}=23.242 ; P<0.000 I\right)$. ** $P<0.0$ I by Tukey/Kramer's post hoc test.
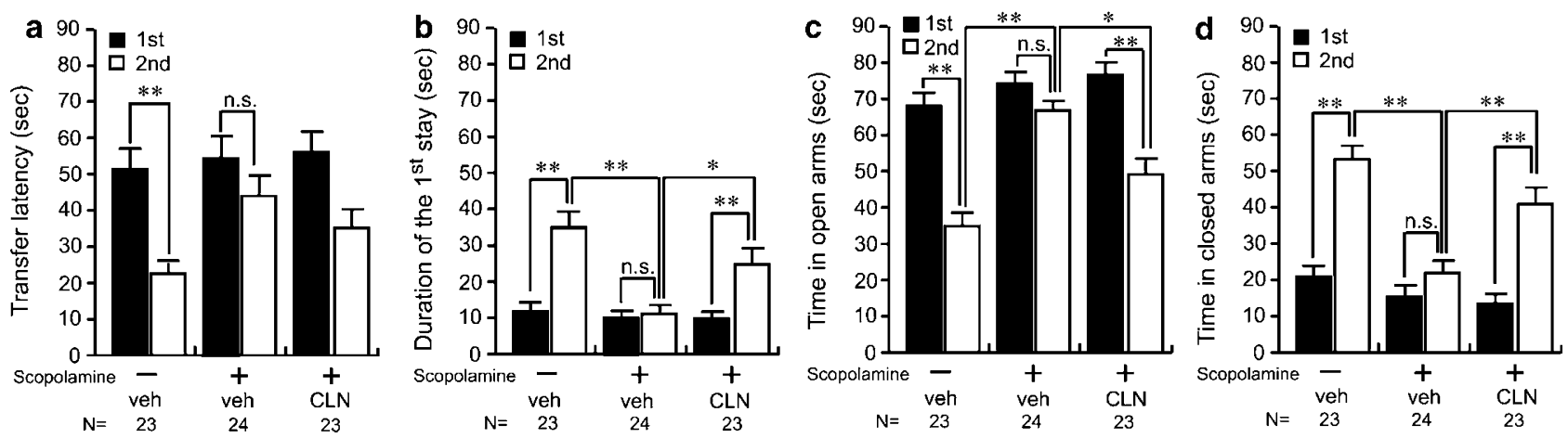

Figure 3 Effect of i.n. administered Colivelin (CLN) on a cholinotoxin model in an elevated-plus maze (EPM). Effect of i.n. administered CLN was examined in EPM, which assesses fear-motivated spatial learning. Scopolamine (I.0 mg/kg s.c.) was administered 30 min before the first session. Intranasal administration of vehicle (veh) or CLN (I nmol per administration) was performed twice as depicted in Figure 2a. The closed column shows results in the first session and the open column shows those in the second session. Data are shown as means \pm SEM. ANOVA revealed a significant effect of treatments on the examined parameters: transfer latency into a closed arm $\left(F_{(5.134)}=5.761 ; P<0.000 \mathrm{I}\right)$ (a, 'transfer latency'), duration of the first stay in the closed arm $\left(F_{(5,134)}=12.168 ; P<0.000 \mathrm{I}\right)$ (b, 'first stay'), cumulative time spent in the open arms $\left(F_{(5,134)}=23.058 ; P<0.000 \mathrm{I}\right)$ (c, 'time in open arms'), and cumulative time spent in the closed arms $\left(F_{(5,134)}=23.027 ; P<0.000 \mathrm{I}\right)$ ( $\mathrm{d}$, 'time in closed arms'). $* P<0.05$, $* * P<0.0 \mathrm{I}$ by Tukey/Kramer's post hoc test. 'n.s.' means not significant.

were no differences in the entry latencies among the four treatment groups (Figure $4 \mathrm{~b}$ ). In the retention trial, the entry latency of the mice receiving s.c. saline and i.n. vehicle administration (control mice) was robustly prolonged compared to that in the acquisition trial, suggesting that the control mice acquired contextual memory in PA task on day 1 and that the memory was retained at least for $24 \mathrm{~h}$. The entry latency in the mice receiving s.c. scopolamine and i.n. vehicle administration in the retention trial was slightly prolonged compared to that in the acquisition trial but was significantly shorter than that in the retention trial of the control mice, indicating that scopolamine impaired memory function. CLN treatment significantly attenuated scopolamine-induced decrease in the entry latency in the retention trial (Figure $4 \mathrm{~b}$ ), suggesting that CLN suppresses scopolamine-induced memory deficits in PA. There was no significant difference in the entry latency between mice receiving s.c. saline/i.n. CLN treatment and mice receiving s.c. saline/i.n. vehicle treatment.
We further performed an RAM task to assess memory performances; acquisition of reference memory and spatial working memory. Low-dose scopolamine $(0.2 \mathrm{mg} / \mathrm{kg} /$ day $)$ or saline were daily injected to mice and their memory performances were evaluated by the task once a day for 6 consecutive days. Their memory performances were further tested by the task for additional 2 days without the injection of scopolamine or saline to monitor the disappearance of the scopolamine's effect. CLN or vehicle was daily i.n. administered for 8 days. Total times required for the mice receiving s.c. saline and i.n. vehicle treatment (saline/vehicle group) to finish the task were significantly shortened and reached a plateau on the fourth day of the trial (day 4) (Figure 5b). Such learning pattern in the total time was similarly observed in another parameter of the test, error percentage in the first eight entries (Figure 5c). Total times required for the mice receiving s.c. saline and i.n. CLN (saline/CLN group) to finish the task were mostly similar to those for the saline/vehicle group, while those required for 
the mice receiving s.c. scopolamine and i.n. vehicle (scopolamine/vehicle group) were not shortened until day 8 , indicating that acquisition of reference memory was completely blocked by scopolamine treatment. Error percentages in the scopolamine/vehicle group were larger than those of the saline/vehicle group day 1 through day 7 (statistically significant on days $1,4,5,6$, and 7), indicating that scopolamine treatment also impaired spatial working
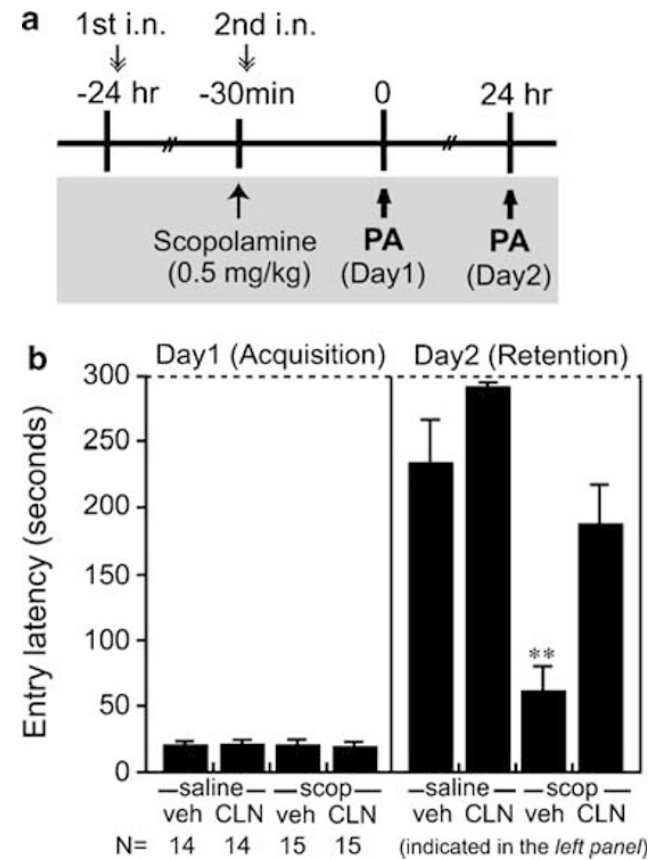

Figure 4 Effect of i.n. administered Colivelin (CLN) on a cholinotoxin model in a passive avoidance (PA) task. The design of the experiment is depicted in (a). We examined the effect of i.n. administered CLN on scopolamine (0.5 mg/kg, s.c.)-induced memory impairment in PA. Entry latencies in the acquisition trial and the retention trial were measured (b). Data are shown as means \pm SEM. ANOVA revealed a significant group difference $\left(F_{(3,54)}=17.897 ; P<0.0001\right)$. $* * P<0.01$ (vs saline plus vehicle, saline plus CLN, or scopolamine plus CLN group) by Tukey/Kramer's post hoc test. memory. Note that both parameters in the scopolamine/ vehicle group returned to the levels equivalent to those of the saline/vehicle group on day 8,2 days after the last injection of scopolamine. Intranasal CLN treatment showed substantial rescue effects on scopolamine-induced memory impairment: shortened total times day 2 through day 7 and decreased error percentages on days 6 and 7 (Figure $5 \mathrm{~b}$ and c), indicating that i.n. CLN treatment inhibited both working and reference memory impairment caused by scopolamine.

\section{CLN's Activity is Stronger Than ADNF or AGA-(C8R)HNG17 In Vivo}

Colivelin has been reported to exhibit its neuroprotective activity by activating the downstream prosurvival signals of putative ADNF receptor and HN receptor in vitro (Chiba et al, 2005; Matsuoka et al, 2006). To delineate the relative in vivo potency of CLN and its component peptides, we compared the effect of i.n. administered CLN with those of its component peptides, ADNF and AGA-(C8R)HNG17 on working memory impairment induced by another cholinotoxin, 3-QNB (Figure 6a). As expected from the results in Figure $2 \mathrm{~b}$, short-term i.n. administration of CLN again significantly attenuated memory impairment caused by $3-$ QNB in a dose-responsive fashion (Figure 6c). Note that i.n. administration of $1 \mathrm{nmol}$ CLN exhibited more potent effect than donepezil $(10 \mathrm{mg} / \mathrm{kg})$. In contrast, short-term i.n. administration of neither ADNF nor AGA-(C8R)HNG17 inhibited 3-QNB-induced memory impairment at all (Figure 6d and e), indicating that only CLN showed some pharmacological effect, unlike its component peptides in vivo.

Activity-dependent neurotrophic factor has been reported to exhibit a neuroprotective effect on memory impairment induced by another cholinotoxin, AF64A, when i.n. administered daily for longer than 1 week (Gozes et al, 2000). Combined with the negative finding shown in Figure 6d, that short-term administration of ADNF did not show any pharmacological effect on cholinotoxin-

\section{a}

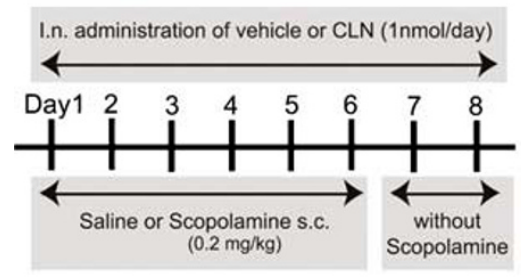

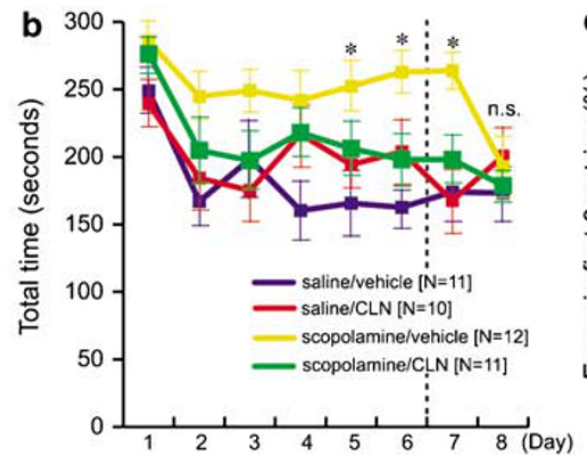

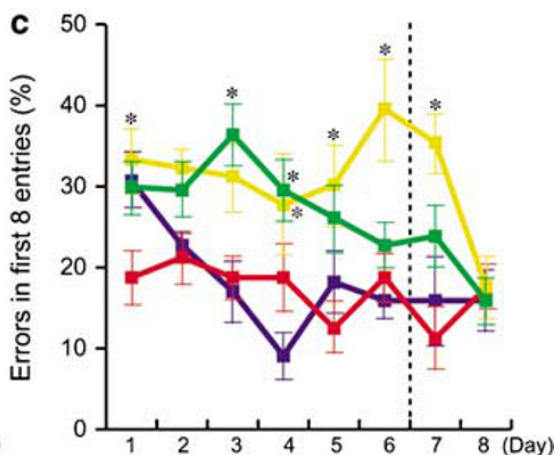

Figure 5 Effect of i.n. administered Colivelin (CLN) on a cholinotoxin model in an radial-arm maze (RAM). The design of the experiment is depicted in (a). We examined the effect of i.n. administered CLN on scopolamine $(0.2 \mathrm{mg} / \mathrm{kg}$, s.c.)-induced memory impairment in RAM. Examined parameters are total time spent in choosing all arms (b, 'total time') and working memory error percentage in the first eight entries (c, 'error\% in the first eight entries', defined as the percentage of overlapped entries in the first eight entries). Saline s.c. plus vehicle i.n. ('control mice', $N=\mid \mathrm{I}$ ), blue line; saline s.c. plus $C L N$ i.n. ( $N=\mid \mathrm{O}$ ), red line; scopolamine s.c. plus vehicle i.n. $(N=12)$, yellow line; scopolamine s.c. plus CLN i.n. $(N=1$ I), green line. Data are shown as means $\pm S E M$. Two-way repeated-measures ANOVA was performed on 'total time' $\left(F_{(3,40)}=9.62 \mathrm{I} ; P<0.000 \mathrm{I}\right.$ for groups, $F_{(7,40)}=5.896 ; P<0.000 \mathrm{I}$ for days, and $F_{(21,40)}=0.922$; $P=0.5627$ for interactions between groups and days) and 'error\%' $\left(F_{(3,40)}=16.602 ; P<0.0001\right.$ for groups, $F_{(7,40)}=3.579 ; P=0.001$ for days, and $F_{(21,40)}=1.736 ; P=0.0254$ for interactions between groups and days). Tukey/Kramer's post hoc test was performed for multiple group comparison $(* P<0.05$, vs the control mice). 
a short-term treatment protocol

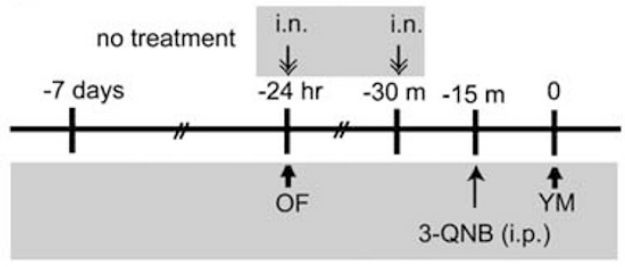

b long-term treatment protocol

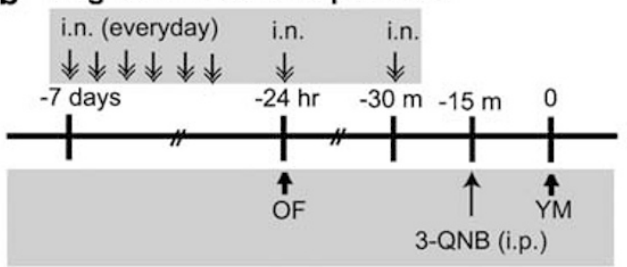

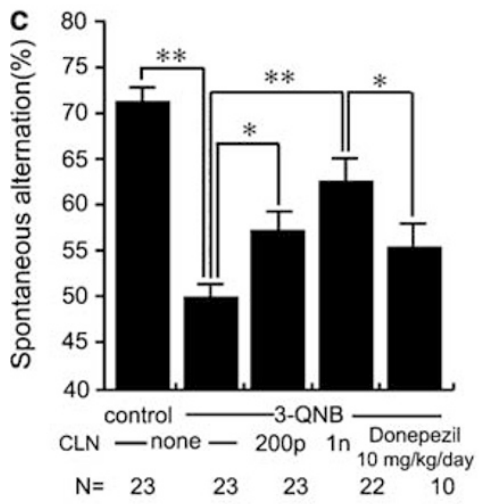
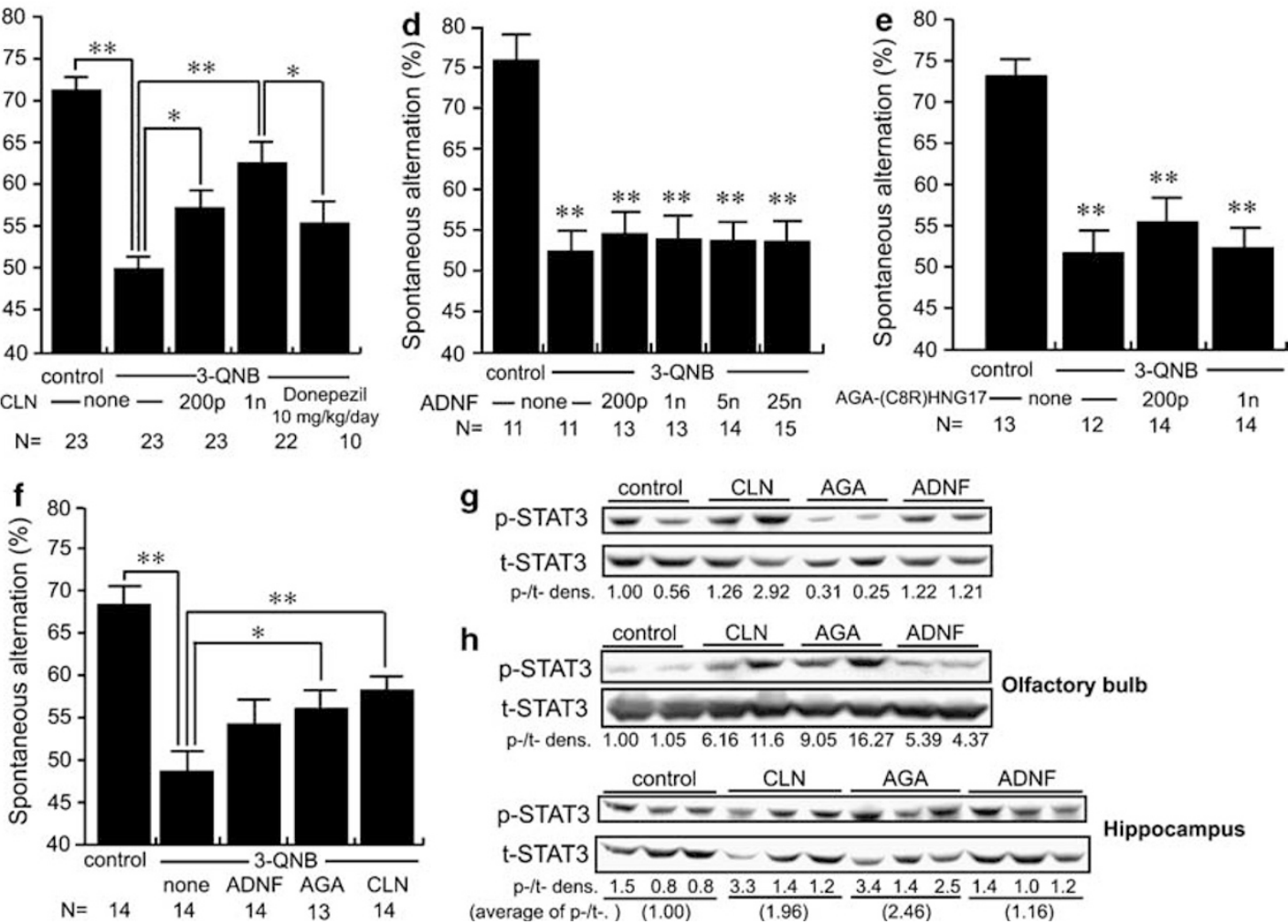

Figure 6 Characterization of Colivelin (CLN)-mediated memory improvement. Experimental designs for short-term and long-term peptide treatments on 3-quinuclidinyl benzilate (3-QNB, $0.5 \mathrm{mg} / \mathrm{kg}$, i.p.)-induced memory impairment are depicted ( $\mathrm{a}$ and b). Effect of short-term i.n. administration of CLN (c), activity-dependent neurotrophic factor (ADNF) (d), or AGA-(C8R)HNGI7 (e) at indicated doses per application in a cholinotoxin (3-QNB) model was examined. Donepezil $(10 \mathrm{mg} / \mathrm{kg}$ ) was i.p. administered as a positive control. High hydrophobicity of AGA-(C8R)HNG I7 prevented us from examining its effect at higher doses. Spontaneous alternation percentage (SA\%) of each treatment group is shown as means \pm SEM. One-way ANOVA revealed significant effects of treatments on SA\%: $F_{(4,63)}=11.817 ; P<0.0001$ for $(c), F_{(5,71)}=6.1 I 3 ; P<0.000$ I for $(d)$, and $F_{(4,48)}=9.488 ; P<0.000$ I for (e). * $P<0.05$, $* * P<0.0$ I by Tukey/Kramer's post hoc test (for (d) and (e), ** $P<0.0$ I vs control). Effect of long-term i.n. administration of the peptides was also examined (f) (ANOVA: $F_{(4,64)}=8.559, P<0.000$ I). $* P<0.05$, $* * P<0.0$ I by Fisher's PLSD multiple comparison test. Immunoblot analysis of the lysates from olfactory bulbs of the mice treated with short-term i.n. administration of vehicle (control), CLN, ADNF, or AGA-(C8R)HNGI7 (AGA) (two lanes each), was carried out with antibodies against both phosphorylated (p-) and total (t-) STAT3 (g). Immunoblot analysis of the brain lysates derived from olfactory bulbs and hippocampuses of the mice treated with long-term i.n. administration was also carried out with antibodies against both p-STAT3 and t-STAT3 (h). Lysates were sampled $30 \mathrm{~min}$ after the second i.n. treatment of the peptides. Values indicated under panels for immunoblot analysis are the densitometrically calculated ratios of p-STAT3 to t-STAT3 expression levels.

mediated memory impairment, it is speculated that repetitive administration of ADNF may increase its efficacy, presumably because it increases the concentrations of ADNF in vivo. Based on this speculation, we administered ADNF, AGA-(C8R)HNG17, or CLN to the mice once a day for 1 week at a dose of $1 \mathrm{nmol}$ per treatment (long-term treatment) to potentiate the effects of the peptides, as depicted in Figure $6 \mathrm{~b}$. We then found that long-term i.n. administration of CLN or AGA-(C8R)HNG17 showed significant inhibition of memory impairment caused by 3-QNB while that of ANDF appeared to show a marginal but statistically insignificant inhibition of it (Figure 6f). Thus, it is likely that the efficacy of i.n. treatment of the peptides can be potentiated by increasing administration frequency.

\section{STAT3 is Involved in CLN-Mediated Inhibition of Cholinotoxin-Induced Memory Impairment}

We further examined levels of STAT3 $\left(\mathrm{Tyr}^{705}\right)$ phosphorylation, which represents activated STAT3 levels, in the brains of mice i.n. administered with CLN, ADNF, or AGA(C8R)HNG17 because it has been shown that STAT3 is an essential effecter for intracellular signals activated by $\mathrm{HN}$ and its derivatives in vitro (Chiba et al, 2005; Hashimoto et al, 2005; Matsuoka et al, 2006). Short-term i.n. administration of CLN tended to increase the STAT3 phosphorylation levels in the olfactory bulb while neither that of ADNF nor AGA-(C8R)HNG17 did so (Figure 6g). We also found that long-term i.n. administration of AGA- 
(C8R)HNG17 as well as that of CLN significantly induced STAT3 phosphorylation in both the olfactory bulb and the hippocampus, indicating that STAT3 phosphorylation levels correlated positively with SA\% obtained in YM. Therefore, we speculate that the pharmacological effect exhibited by the peptides in vivo was at least partially due to STAT3 activation (Figure 6h).

\section{Intranasal Administration of CLN Protects the Cholinergic Systems from Toxicity by A $225-35$}

Intracerebroventricular injection of toxic $\mathrm{A} \beta$ peptides has been reported to impair spatial working memory in rodents (Maurice et al, 1996; Yamada et al, 1999, 2005). We have recently established a toxic $\mathrm{A} \beta$-induced mouse model of $\mathrm{AD}$ by using repetitive i.c.v. injections of a small amount of toxic $\mathrm{A} \beta$ via a cannula implanted into the lateral ventricle (Chiba et al, 2005; Yamada et al, 2005). Using this model, we examined the therapeutic effect of i.n. administered CLN on $\mathrm{A} \beta$ toxicity in vivo. We i.c.v. injected $1 \mathrm{nmol}$ of the $\mathrm{A} \beta 25-35$ peptide every other day for 3 weeks (totally 10 times) together with or without i.n. administration of $1 \mathrm{nmol}$ CLN once a day for 3 weeks, as schematically shown in Figure 7 a. Repetitive A $\beta 25-35$ injection efficiently decreased SA\% compared to the vehicle-injected controls (Figure $7 \mathrm{~b}$ ). SA\% of $\mathrm{A} \beta$-injected mice with i.n. CLN treatment was almost equal to that of the water-injected control mice, suggesting that i.n. administration of CLN completely suppressed memory impairment induced by repetitive i.c.v. injection of toxic $A \beta$ (Figure $7 b$ ). To confirm the effect of i.n. CLN treatment on $\mathrm{A} \beta$-induced memory impairment, we also performed PA (Figure 7c). In the retention trial, $\mathrm{A} \beta$ injection significantly reduced entry latency into the darkened chamber and i.n. CLN treatment inhibited A $\beta$ induced reduction of entry latency, indicating that i.n. CLN treatment attenuated contextual memory impairment caused by $\mathrm{A} \beta$ detected in PA as well as $\mathrm{A} \beta$-induced spatial working memory impairment in YM.

As reported in earlier studies (Yamada et al, 1999, 2005), i.c.v. injection of $A \beta$ downregulates the expression level of ChAT in neurons of the medial septum. There is a positive correlation between the extent of spatial working memory impairment and the decrease in numbers of ChAT-positive neurons in the medial septum. By IHC analysis of the medial septa of $\mathrm{A} \beta 25$-35-injected mice with or without i.n. administration of CLN (Figure 7d and e), we observed a significant decrease in the numbers of ChAT-immunoreactive neurons in the medial septa of $\mathrm{A} \beta 25$-35-injected mice (232.3 \pm 16.0 ChAT-positive neurons), compared to the controls $(555.6 \pm 57.4, P<0.01)$ (Figure $7 \mathrm{e}$ ). In accordance with the SA\% in YM, there were nearly as many ChATpositive neurons in $\mathrm{A} / 25$-35-injected mice with i.n. administration of CLN $(514.0 \pm 21.1)$ as those in the controls, suggesting that i.n. CLN treatment blocked the decrease in the number of ChAT-positive neurons, caused by repetitive i.c.v. injection of $\mathrm{A} \beta 25-35$ (Figure 7e).

To elucidate the mechanism underlying CLN-mediated inhibition of $\mathrm{A} \beta 25$-35-induced decrease in the number of ChAT-positive neurons, we next examined mRNA expression of ChAT and GAPDH in PC12 cells treated with $100 \mathrm{pM}$ CLN, ADNF, or AGA-(C8R)HNG17 by semiquantitative RTPCR (Figure 7f). We then found a significant increase in the
mRNA expression levels of ChAT in PC12 cells treated with 100 pM CLN compared to those of the control cells. mRNA expression levels of ChAT were also upregulated by $100 \mathrm{pM}$ AGA-(C8R)HNG17, but not by 100 pM ADNF. CLN and AGA-(C8R)HNG17 treatment, but not ADNF treatment, also increased mRNA expression levels of vesicular VAChT as well (Figure 7f). It is reasonable that CLN simultaneously upregulates mRNA expression levels of VAChT as well as those of ChAT because mRNA expression of these molecules has been reported to be regulated in the same fashion (Oda, 1999). Note that mRNA expression levels of GAPDH were not affected by any treatment (Figure 7f). Taken together, these results suggest that CLN-induced upregulation of both ChAT and VAChT expressions, which is likely to be due to STAT3 activation, may contribute to CLN-mediated suppression of AD-relevant cholinergic dysfunction.

\section{CLN does not Inhibit AChE Activity}

It is also possible that CLN supports cholinergic neurotransmission by mechanisms other than upregulation of ChAT expression. To investigate whether CLN directly inhibits activity of the acetylcholinestrase (AChE), a key enzyme in neuronal choline degradation and a target of currently used anti-AD drugs, we examined the effect of CLN treatment on the AChE activity by Ellman's method (Figure 8). In the assay, we detected a significant increase in absorbance at $416 \mathrm{~nm}$ when acetylthiocholine, DTNB or Ellman's reagent, and AChE were mixed in vitro. Absorbance at $416 \mathrm{~nm}$ was dose dependently reduced by a $\mathrm{AChE}$ inhibitor tacrine, suggesting that tacrine inhibited AChE activity. In clear contrast, CLN did not inhibit AChE activity.

\section{CLN Treatment did not Cause Obvious Side Effects}

From the standpoint of clinical application of CLN, it is very important to delineate its potential toxic side effects. For that purpose, we preliminarily examined side effects of CLN to liver and kidney by measuring serum ALT (GOT), AST (GPT), and CRE (Figure 9a-c). To assess acute side effects, we injected $200 \mathrm{nmol}$ of CLN i.v. to CD-1 mice and sampled their sera $6 \mathrm{~h}$ after the injection. No significant differences in the three parameters were observed between sera of vehicle-treated control mice and those of CLN-treated mice. Similarly, subacute toxicities of CLN to liver or kidney were not recognized in the sera after i.n. administration of $5 \mathrm{nmol}$ CLN once a day for 1 week. In addition, no behavioral abnormality or apparent health disturbance was documented in daily observation.

\section{DISCUSSION}

We have here established the efficacy of i.n. treatment of CLN against AD-relevant memory impairment. Delivery of peptide drugs into the CNS is always troublesome because BBB blocks their transportation. CLN has the highest potency among the $\mathrm{HN}$ derivatives thus far developed (EC50 is <100 fM) and is therefore effective against AD-relevant memory impairment with i.p. or i.v. administration, which enables a very small fraction of CLN to enter the CNS (Chiba et al, 2005). It is apparent, however, that a 
a
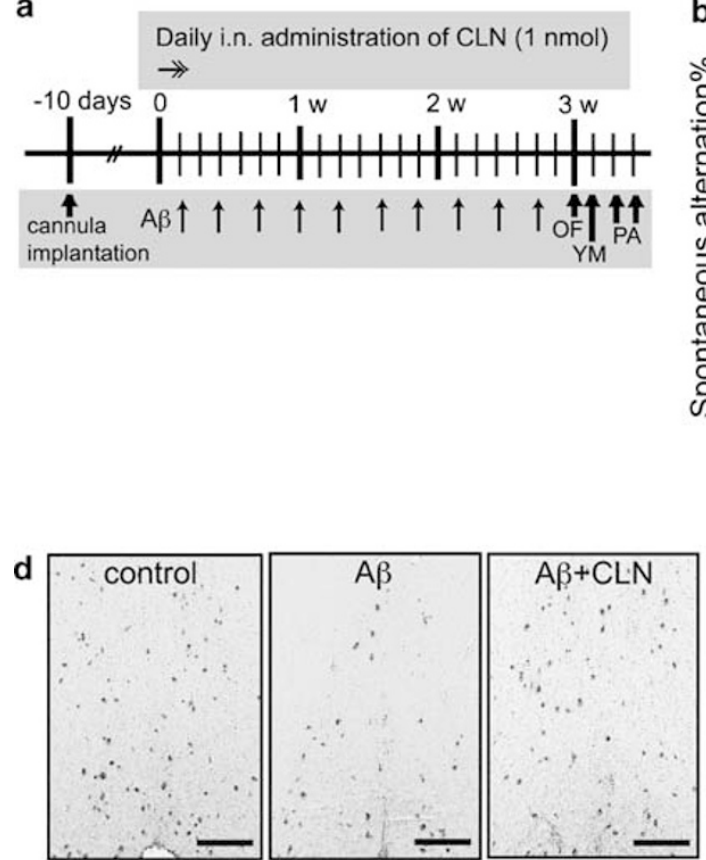
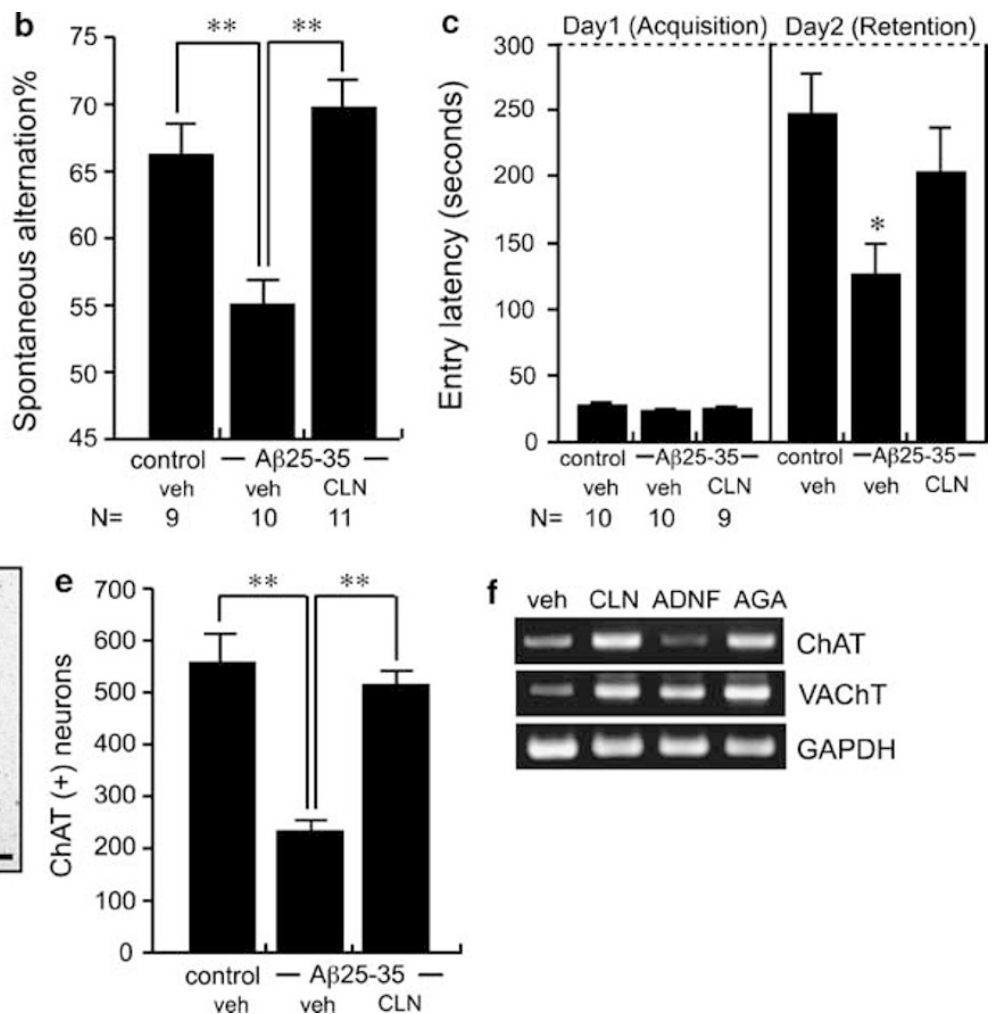

f

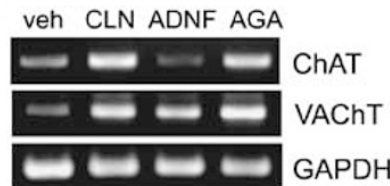

Figure 7 Effect of i.n. Colivelin (CLN) treatment on the repetitive $A \beta$ injection model. We examined the effect of i.n. administered CLN on $A \beta 25-35$ induced working memory impairment. The experimental design is depicted in (a). Spontaneous alternation percentage of mice with repetitive i.c.v. injection of sterile water or A $\beta 25-35$ together with i.n. administration of vehicle (veh) or CLN (I nmol per application) is shown as means \pm SEM (b). One-way ANOVA revealed a significant effect of treatments $\left(F_{(2.44)}=|4.9| 7 ; P<0.000 I\right)$. ** $P<0.01$ by Tukey/Kramer's post hoc test. Passive avoidance test (PA) was also performed to examine the effect of i.n. CLN on memory impairment caused by repetitive $A \beta$ i.c.v. administration (c). Entry latencies, an index of contextual memory in PA, are shown as means \pm SEM (ANOVA: $F_{(2,26)}=4.775 ; P=0.017 I$ ). $* P<0.05$ by Dunnett's post hoc test (vs control). Brain sections of the medial septum were immunohistochemically stained with anti-choline acetyltransferase (ChAT) antibody (d; bar: $100 \mu m$ ). ChAT-immunoreactive neurons in the medial septum were counted and compared among the groups (e). Data are shown as means \pm SEM (*** $<0.0$ I). (f) RT-PCR amplification of ChAT, vesicular acetylcholine transporter (VAChT), and glyceraldehyde-3-phosphate dehydrogenase (GAPDH) mRNAs was performed using PCI2 cells stimulated for $24 \mathrm{~h}$ with peptides (lanes: vehicle, veh; 100 pM Colivelin, CLN; 100 pM activity-dependent neurotrophic factor (ADNF), ADNF; I00 pM AGA-(C8R)HNG I7, AGA).

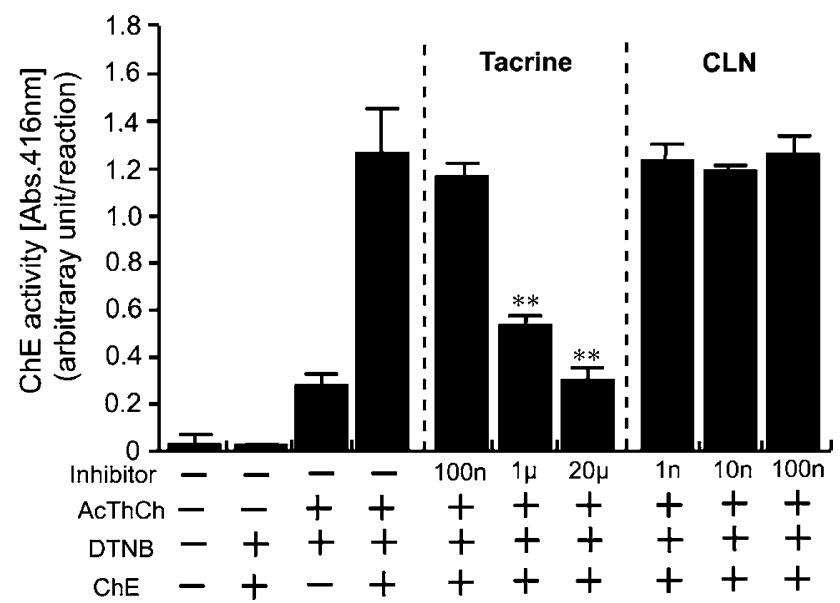

Figure 8 Effect of Colivelin (CLN) on acetylcholinesterase (AChE) activity. We measured AChE activity with or without a AChE inhibitor tacrine or CLN at indicated concentrations by Ellman's method $(N=3)$. Data are shown as means \pm SD $(* * P<0.0 I)$. AcThCh, acetylthiocholine; DTNB, 5,5'-dithio-bis-(2-nitrobenzoic acid).

large amount of potent $\mathrm{HN}$ derivatives in the systemic blood stream by i.p. or i.v. administration causes side effects in extra-CNS organs. Furthermore, given the long clinical course of $\mathrm{AD}$ patients, anti-AD drugs should be noninvasively administered. Accordingly, we searched for drug delivery systems more selective and less invasive than i.c.v., i.v., or i.p. administrations. We have here found that i.n. administration may match these conditions.

We tried to prove the entrance of CLN into the CNS. It is true that we do not have direct evidence that intact CLN reached CNS by i.n. administration. IHC analysis, however, indicated that CNS areas specifically immunoreactive to two CLN antibodies recognizing distinct epitopes (ADNF and AGA-(C8R)HNG17 portions) of CLN were largely overlapped (Figure 1). Combined with another finding by behavioral tests that i.n. ADNF or i.n. AGA-(C8R)HNG17 has by far weaker activity than i.n. CLN against memory impairment (Figure $6 \mathrm{c}-\mathrm{e}$ ), it is reasonable to think that intact CLN, but not a mixture of degraded peptides containing $\mathrm{ADNF}$ or $\mathrm{HN}$ epitope, reached CNS and improved memory impairment. As reported in earlier studies (Illum, 2000; Thorne et al, 2004), CLN immunoreactivity was predominantly detected in the medial and ventral regions of the olfactory bulbs of the mice with i.n. CLN treatment (Figure $1 \mathrm{a}$ and $\mathrm{b}$ ), suggesting that i.n. administered CLN was likely to be delivered to CNS by mechanisms called the dendritic transporting processes of the olfactory sensory neurons (Illum, 2000; Thorne et al, 

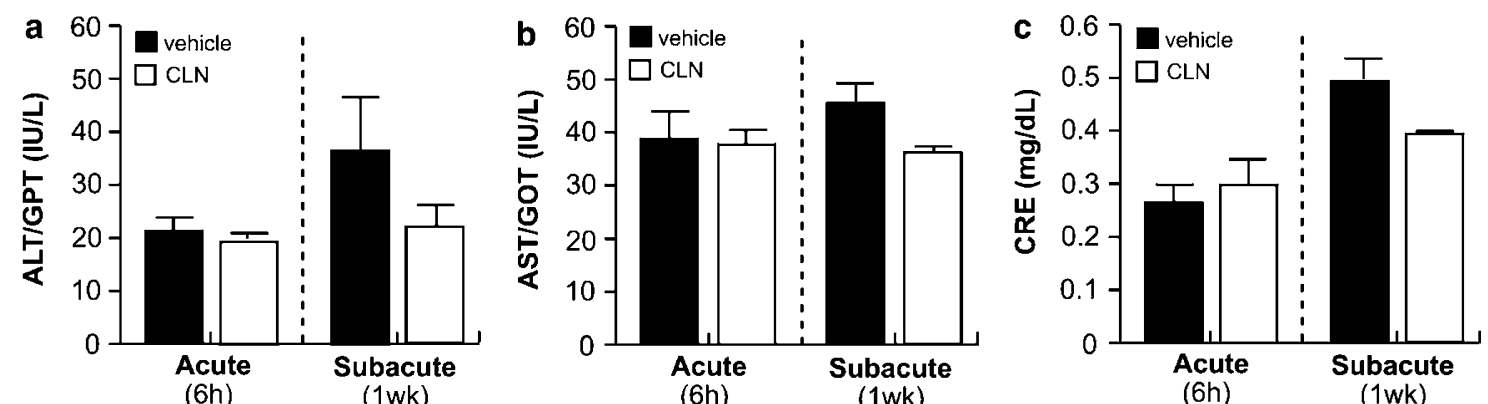

Figure 9 Estimation of acute and subacute toxicities of Colivelin (CLN) monitored by serum markers. To examine acute and subacute toxicities of CLN to liver and kidney, we measured serum alanine aminotransferase (ALT/GPT) (a), aspartate aminotransferase (AST/GOT) (b), and creatinin (CRE) (c, N=3 each). To monitor acute toxicities, we i.v. injected $200 \mathrm{nmol}$ of CLN to CD-I mice at 8 weeks of age and collected sera $6 \mathrm{~h}$ after the treatment. To monitor subacute toxicities, we i.n. administered CLN ( 5 nmol per application) once a day for I week, followed by serum collection. Data are shown as means \pm SD Standard values measured in normal CD-I mice at 31 weeks of age are $33 \pm 7$ for ALT/GPT, $49 \pm 5$ for AST/GOT, and $0.31 \pm 0.03$ for CRE, respectively (CLEA Japan Inc.).

2004). Although the detailed mechanisms underlying noseto-brain transport remain elusive, they are thought to consist of both an extracellular transportation system, by which it may take several minutes for peptides to reach the CNS, and an intracellular transportation system, by which it may take more than $6 \mathrm{~h}$ for peptides to reach the CNS, based on previous observations by electron microscopy (Broadwell and Balin, 1985; Balin et al, 1986). We also observed substantial immunoreactivity around the optic chiasms. Combined with the previous finding that the trigeminal nerve is another route for peptides to the CNS (Illum, 2000; Thorne et al, 2004), we speculate that the optic nerve may also serve as a route.

We also compared the efficacy of i.n. administration of CLN with two other drug delivery routes, i.p. and s.c. (Figure 2d). We observed some pharmacological effect of CLN by i.n. administration on scopolamine-induced memory impairment, confirming that i.n. administration is a highly specific method of delivering peptide to the CNS. It is, however, important to confirm that the efficacy of i.n. administration is not limited to rodents. The routes from the nasal cavity to CNS are thought to remain in humans although human olfactory nervous systems are relatively degenerated compared with those of rodents. In support of the issue, i.n. administration of insulin in humans has been reported to improve memory without obvious systemic side effects such as hypoglycemia (Benedict et al, 2004). Future investigation on the pharmacodynamics of CLN is warranted.

Colivelin, consisting of ADNF and AGA-(C8R)HNG17, exhibits its neuroprotective activity by activating both ADNF receptor- and HN receptor-mediated prosurvival pathways in vitro (Chiba et al, 2005; Matsuoka et al, 2006). Both HN derivatives and ADNF-related peptides have also been reported to attenuate cholinotoxin-induced memory impairment in vivo (Gozes et al, 2000; Chiba et al, 2005). We found that short-term i.n. CLN treatment ameliorated the AD-relevant memory disturbance while short-term i.n. treatment with the individual components of CLN at the same dose did not reproduce the pharmacological effect of CLN (Figure 6c-e). The results indicate that i.n. administration of CLN exhibits a more potent pharmacological effect than those of ADNF or AGA-(C8R)HNG17 in vivo, presumably because the concentration of CLN is more easily elevated by i.n. administration to its effective level in CNS than those of its component peptides. In accordance with this idea, we also found that long-term i.n. administration of AGA-(C8R)HNG17 significantly and that of ADNF marginally attenuated memory impairment caused by 3-QNB (Figure 6f), suggesting that repetitive i.n. administration increases the efficacy of the neuroprotective peptides by increasing intracranial concentrations. In addition, the fusion of AGA-(C8R)HNG17 to ADNF (CLN) may enhance the in vivo ADNF's rescue effect on ALS model mice (Chiba et al, 2004, 2006). Taken together, it is possible that the ADNF portion of CLN may contribute to the pharmacological effect of CLN on AD model mice.

The targets of CLN remain enigmatic because neither the receptor of ADNF nor that of $\mathrm{HN}$ has been identified. Recently, it has been reported that ADNF binds to and stabilizes tubulin to protect cells from various types of cellular stress (Holtser-Cochav et al, 2006). ADNF-related peptide, NAP (NAPVSIPQ) also specifically binds to tubulin at an NAP-binding site as ADNF does, which may explain, in part, the broad neuroprotective activity offered by these peptides. In this paradigm, ADNF should pass through the plasma membrane. However, we have no evidence so far that CLN, a 26-amino-acid peptide, also physically goes through the plasma membrane and binds to tubulin. Our in vitro observation suggested that activation of the $\mathrm{Ca}^{2+} /$ calmodulin-dependent protein kinase IV (CaMKIV) is essential in the neuroprotective effect of ADNF although the precise mechanism remains uncharacterized (Chiba et al, 2004, 2005). The involvement of tubulin and CaMKIV in the pharmacological effect of CLN in vivo remains to be addressed.

The HN receptor is thought to be on the cell membrane (Hashimoto et al, 2001a, b; Nishimoto et al, 2004; Matsuoka et al, 2006) although it remains unidentified. Our recent studies have demonstrated that $\mathrm{HN}$ signals against $\mathrm{AD}$ relevant neuronal death are intracellularly mediated by STAT3 in vitro (Chiba et al, 2005; Hashimoto et al, 2005). Earlier studies established the therapeutic effect of HN derivatives on functional memory deficits caused by $\mathrm{AD}$-relevant insults, such as cholinotoxins and soluble $\mathrm{A} \beta$ (Mamiya and Ukai, 2001; Krejcova et al, 2004; Chiba et al, 2005). It has long been unclear, however, how HN derivatives exert their effects in vivo. In this study, we 
observed upregulation of STAT3 phosphorylation levels only in the olfactory bulbs of mice with i.n. CLN treatment when they were treated with the short-term i.n. protocol (Figure $6 \mathrm{~g}$ ), suggesting that elevation of STAT3 phosphorylation levels may be correlated with the pharmacological effect of CLN. This hypothesis was further supported by another observation, that STAT3 phosphorylation levels in the olfactory bulbs and the hippocampuses were upregulated in mice with i.n. administration of CLN or AGA(C8R)HNG17 under the long-term protocol (Figure 6h). Additional detailed mechanisms underlying the pharmacological effect of CLN remain to be addressed in future investigation.

Given that HN derivatives significantly antagonize cholinotoxin-induced memory impairment, it is natural to think that they directly affect the cholinergic systems. In support of this idea, i.n. administered CLN inhibited the downregulation of ChAT expression in the medial septa by $\mathrm{A} \beta$ treatment (Figure $7 \mathrm{~d}$ and $\mathrm{e}$ ), consistent with our previous finding that i.c.v. injection of CLN antagonizes $\mathrm{A} \beta$-induced downregulation of ChAT expression (Chiba et al, 2005). We further found that mRNA expression of ChAT and VAChT in PC12 cells was upregulated by treatment with CLN or AGA-(C8R)HNG17 (Figure 6e). Together with an earlier finding that leukemia inhibitory factor, which activates STAT3, maintains ChAT expression (Cheema et al, 1998); it is suggested that activation of STAT3 transcriptionally upregulates ChAT and VAChT expression. Considering that ChAT catalyzes ACh synthesis and VAChT uptakes ACh to synaptic vesicles (Oda, 1999), we speculate that CLN-mediated STAT3 activation increases presynaptic ACh storage and supports cholinergic neurotransmission.

In addition, it is reported that STAT3 may bind to the $5^{\prime}$ upstream region of the presenilin 1 (PS1) gene, a causative gene of FAD, and regulate its transcription (Rogaev et al, 1997). Given that PS1 is a major component of the $\gamma$-secretase that cleaves amyloid precursor protein and contributes to the generation of $\mathrm{A} \beta$ (Takasugi et al, 2003), activation of STAT3 might affect memory impairment related to $A D$ via transcriptional regulation of PS1.

In summary, we provide the first evidence that $\mathrm{HN}$ derivatives can be i.n. administered for treatment of AD. Especially, i.n. CLN treatment shows great promise in development of a novel AD therapy. We also demonstrate that HN derivatives may trigger STAT3-mediated signals in vivo as well as in vitro, which may consequently increase presynaptic ACh storage by transcriptionally upregulating ChAT and VAChT expression and promote cholinergic neurotransmission.

\section{ACKNOWLEDGEMENTS}

We thank Dr Ikuo Nishimoto, who initiated the work and unfortunately passed away on 17 October 2003. We are indebted to Drs Yasuo Ikeda and Masaki Kitajima for essential help. We also thank Dr Etsuro Ogata, and $\mathrm{Mr}$ Yoshiomi and Mrs Yumi Tamai for indispensable support; Dr Dovie Wylie for expert assistance; Ms T Hiraki, $\mathrm{H}$ Matsumoto, $\mathrm{T}$ Yoshida-Nishimoto, and S Uchida for expert assistance; and all members of the Department of
Anatomy for essential cooperation. This work was supported in part by grants from the Japan Society for the Promotion of Science, the Program for Promotion of Fundamental Studies in Health Sciences of the National Institute of Biomedical Innovation (NIBIO), the KEIO Gijuku Academic Development Funds (TC), and in part by Noevir Co., Ltd.

\section{DISCLOSURE/CONFLICT OF INTEREST}

The authors declare that except for income received from our primary employer no financial support or compensation has been received from any individual or corporate entity over the past 3 years for research or professional service and there are no personal financial holdings that could be perceived as constituting a potential conflict of interest.

\section{REFERENCES}

Bales KR, Tzavara ET, Wu S, Wade MR, Bymaster FP, Paul SM et al (2006). Cholinergic dysfunction in a mouse model of Alzheimer disease is reversed by an anti-A beta antibody. J Clin Invest 116: 825-832.

Balin BJ, Broadwell RD, Salcman M, El-Kalliny M (1986). Avenues for entry of peripherally administered protein to the central nervous system in mouse, rat, and squirrel monkey. J Comp Neurol 251: 260-280.

Bartus R, Dean III RL, Beer B, Lippa AS (1982). The cholinergic hypothesis of geriatric memory dysfunction. Science 217: 408-417.

Benedict C, Hallschmid M, Hatke A, Schultes B, Fehm HL, Born J et al (2004). Intranasal insulin improves memory in humans. Psychoneuroendocrinology 29: 1326-1334.

Brenneman DE, Gozes I (1996). A femtomolar-acting neuroprotective peptide. J Clin Invest 97: 2299-2307.

Brenneman DE, Hauser J, Neale E, Rubinraut S, Fridkin M, Davidson A et al (1998). Activity-dependent neurotrophic factor: structure-activity relationships of femtomolar-acting peptides. J Pharmacol Exp Ther 285: 619-627.

Broadwell RD, Balin BJ (1985). Endocytic and exocytic pathways of the neuronal secretory process and trans-synaptic transfer of wheat germ agglutinin-horseradish peroxidase in vivo. J Comp Neurol 242: 632-650.

Caccamo A, Oddo S, Billings LM, Green KN, Martinez-Coria H, Fisher A et al (2006). M1 receptors play a central role in modulating AD-like pathology in transgenic mice. Neuron 49: 671-682.

Cheema SS, Arumugam D, Murray SS, Bartlett PF (1998). Leukemia inhibitory factor maintains choline acetyltransferase expression in vivo. Neuroreport 9: 363-366.

Chiba T, Hashimoto Y, Tajima H, Yamada M, Kato R, Niikura T et al (2004). Neuroprotective effect of activity-dependent neurotrophic factor against toxicity from familial amyotrophic lateral sclerosis-linked mutant SOD1 in vitro and in vivo. J Neurosci Res 78: 542-552.

Chiba T, Yamada M, Hashimoto Y, Sato M, Sasabe J, Kita Y et al (2005). Development of a femtomolar-acting humanin derivative named colivelin by attaching activity-dependent neurotrophic factor to its $\mathrm{N}$ terminus: characterization of colivelin-mediated neuroprotection against Alzheimer's disease-relevant insults in vitro and in vivo. J Neurosci 25: 10252-10261.

Chiba T, Yamada M, Sasabe J, Terashita K, Aiso S, Matsuoka M et al (2006). Colivelin prolongs survival of an ALS model mouse. Biochem Biophys Res Commun 343: 793-798. 
Coyle JT, Price DL, DeLong MR (1983). Alzheimer's disease: a disorder of cortical cholinergic innervation. Science 216: 1184-1190.

Fisher A, Brandeis R, Pittel Z, Karton I, Sapir M, Dachir S et al (1989). (+)-cis-2-methyl-spiro(1,3-oxathiolane-5,3') quinuclidine (AF102B): a new M1 agonist attenuates cognitive dysfunctions in AF64A-treated rats. Neurosci Lett 102: 325-331.

Gozes I, Bardea A, Reshef A, Zamostiano R, Zhukovsky S, Rubinraut $S$ et al (1996). Neuroprotective strategy for Alzheimer disease: intranasal administration of a fatty neuropeptide. Proc Natl Acad Sci USA 93: 427-432.

Gozes I, Giladi E, Pinhasov A, Bardea A, Brenneman DE (2000). Activity-dependent neurotrophic factor: intranasal administration of femtomolar-acting peptides improve performance in a water maze. J Pharmacol Exp Ther 293: 1091-1098.

Hashimoto Y, Niikura T, Ito Y, Sudo H, Hata M, Arakawa E et al (2001a). Detailed characterization of neuroprotection by a rescue factor humanin against various Alzheimer's disease-relevant insults. J Neurosci 21: 9235-9245.

Hashimoto Y, Niikura T, Tajima H, Yasukawa T, Sudo H, Ito Y et al (2001b). A rescue factor abolishing neuronal cell death by a wide spectrum of familial Alzheimer's disease genes and A $\beta$. Proc Natl Acad Sci USA 98: 6336-6341.[Erratum in: Proc Natl Acad Sci USA 98: 12854, 2001].

Hashimoto Y, Suzuki H, Aiso S, Niikura T, Nishimoto I, Matsuoka $M$ (2005). Involvement of tyrosine kinases and STAT3 in humanin-mediated neuroprotection. Life Sci 77: 3092-3104.

Holtser-Cochav M, Divinski I, Gozes I (2006). Tubulin is the target binding site for NAP-related peptides: ADNF-9, D-NAP, and D-SAL. J Mol Neurosci 28: 303-307.

Illum L (2000). Transport of drugs from the nasal cavity to the central nervous system. Eur J Pharm Sci 11: 1-18.

Itoh J, Nabeshima T, Kameyama T (1991). Utility of an elevated plus-maze for dissociation of amnesic and behavioral effects of drugs in mice. Eur J Pharmacol 194: 71-76.

Jhoo JH, Kim HC, Nabeshima T, Yamada K, Shin EJ, Jhoo WK et al (2004). Beta-amyloid (1-42)-induced learning and memory deficits in mice: involvement of oxidative burdens in the hippocampus and cerebral cortex. Behav Brain Res 155: 185-196.

Kawasumi M, Chiba T, Yamada M, Miyamae-Kaneko M, Matsuoka M, Nakahara J et al (2004). Targeted introduction of V642I mutation in amyloid precursor protein gene causes functional abnormality resembling early stage of Alzheimer's disease in aged mice. Eur J Neurosci 19: 2826-2838.

Krejcova G, Patocka J, Slaninova J (2004). Effect of humanin analogues on experimentally induced impairment of spatial memory in rats. J Pept Sci 10: 636-639.

Lane RM, Kivipelto M, Greig NH (2004). Acetylcholinesterase and its inhibition in Alzheimer disease. Clin Neuropharmacol 27: 141-149.
Mamiya T, Ukai M (2001). [Gly(14)]-Humanin improved the learning and memory impairment induced by scopolamine in vivo. Br J Pharmacol 134: 1597-1599.

Matsuoka M, Hashimoto Y, Aiso S, Nishimoto I (2006). Humanin and colivelin: neuronal-death-suppressing peptides for Alzheimer's disease and amyotrophic lateral sclerosis. CNS Drug Rev 12: 113-122.

Mattson M (2004). Pathways towards and away from Alzheimer's disease. Nature 430: 631-639.

Maurice T, Lockhart BP, Privat A (1996). Amnesia induced in mice by centrally administered beta-amyloid peptides involves cholinergic dysfunction. Brain Res 706: 181-193.

Nishimoto I, Matsuoka M, Niikura T (2004). Unravelling the role of humanin. Trends Mol Med 10: 102-105.

Oda Y (1999). Choline acetyltransferase: the structure, distribution and pathologic changes in the central nervous system. Pathol Int 49: 921-937.

Petersen RC, Thomas RG, Grundman M, Bennett D, Doody R, Ferris $S$ et al (2005). Vitamin $\mathrm{E}$ and donepezil for the treatment of mild cognitive impairment. $N$ Engl J Med 352: 2379-2388.

Rogaev EI, Sherrington R, Wu C, Levesque G, Liang Y, Rogaeva EA et al (1997). Analysis of the $5^{\prime}$ sequence, genomic structure, and alternative splicing of the presenilin-1 gene (PSEN1) associated with early onset Alzheimer disease. Genomics 40: 415-424.

Tajima H, Niikura T, Hashimoto Y, Ito Y, Kita Y, Terashita K et al (2002). Evidence for in vivo production of humanin peptide, a neuroprotective factor against Alzheimer's disease-related insults. Neurosci Lett 324: 227-231.

Takasugi N, Tomita T, Hayashi I, Tsuruoka M, Niimura M, Takahashi Y et al (2003). The role of presenilin cofactors in the gamma-secretase complex. Nature 422: 438-441.

Thorne RG, Pronk GJ, Padmanabhan V, Frey II WH (2004). Delivery of insulin-like growth factor-I to the rat brain and spinal cord along olfactory and trigeminal pathways following intranasal administration. Neuroscience 127: 481-496.

Winblad B, Kilander L, Eriksson S, Minthon L, Batsman S, Wetterholm AL, et al, Severe Alzheimer's Disease Study Group (2006). Donepezil in patients with severe Alzheimer's disease: double-blind, parallel-group, placebo-controlled study. Lancet 367: 1057-1065.

Yamada K, Tanaka T, Mamiya T, Shiotani T, Kameyama T, Nabeshima $\mathrm{T}$ (1999). Improvement by nefiracetam of $\beta$-amyloid(1-42)-induced learning and memory impairments in rats. Br J Pharmacol 126: 235-244.

Yamada M, Chiba T, Sasabe J, Nawa M, Tajima H, Niikura T et al (2005). Implanted cannula-mediated repetitive administration of Abeta25-35 into the mouse cerebral ventricle effectively impairs spatial working memory. Behav Brain Res 164: 139-146. 